\title{
SOBRE BOURDIEU, EL HABITUS Y LA DOMINACIÓN MASCULINA: TRES APUNTES
}

\author{
Luisa Posada Kubissa \\ Universidad Complutense de Madrid (España) \\ lposada@filos.ucm.es
}

\begin{abstract}
RESUMEN / ABSTRACT
Frente a la naturalización, lo que llama "deshistoricización", Pierre Bourdieu, desde su "estructuralismo constructivista" o su "constructivismo genético", analiza cómo el poder es constitutivo de la sociedad y existe en las cosas y en los cuerpos, incorporándose a los habitus mismos que definen la subjetividad. De este modo, sus análisis abren la puerta a una reconsideración teórico-crítica de las relaciones entre los sexos y, precisamente por ello, son de interés para una perspectiva crítico-feminista, si bien hay que reseñar la escasa atención bourdiueana a la producción de tal perspectiva.
\end{abstract}

PaLABRAS ClAVE: deshistorización, habitus, violencia simbólica, feminismo, Bourdieu.

\section{ABOUT BOURDIEU, HABITUS AND MALE DOMINATION: THREE NOTES}

Opposite to naturalization, what he calls "deshistorization", Pierre Bourdieu, from its "constructivist structuralism" or his"genetic constructivism", examines how power is constitutive of the society and it exists in things and bodies, including itself in the same habitus that define subjectivity. Thus, his analysis opens the door to a critical reconsideration of the relations between the sexes and, precisely for this reason, it is of interest to a critical-feminist perspective, although we must take into account the scant attention Bourdieu gives to such perspective.

KEYWORDS: Deshistorization, habitus, simbolic violence, feminism, Bourdieu.

RT En los años 90, con la desestabilización de Butler de la categoría "mujeres" como sujeto del feminismo (Butler 1990), lo que queda claro, más allá de todo debate, es que el orden heterosexual y androcéntrico responde a una construcción discursiva y de poder. Esta construcción atraviesa la vida social, los cuerpos y las estructuras, presentándose como un orden binario que responde al orden natural de las cosas.

Frente a esta pretendida naturalización, lo que llama "deshistoricización", Pierre Bourdieu, desde su propia posición de un "estructuralismo constructivista" o un "constructivismo genético" (Bourdieu 2007 b: 127)"parte de una doble ontología de lo social. El poder es constitutivo de la sociedad y, ontológicamente, existe en las cosas y en los cuerpos, en los campos y en los habitus, en las instituciones y en 
los cerebros. Por lo tanto, el poder existe físicamente, objetivamente, pero también simbólicamente"(Capdevielle 2011, 32). En palabras del propio Bourdieu: "Por estructuralismo o estructuralista, quiero decir que existen en el mundo social, y no solamente en los sistemas simbólicos, lenguaje, mito, etc., estructuras objetivas, independientemente de la conciencia y de la voluntad de los agentes, que son capaces de orientar o de coaccionar sus prácticas o sus representaciones. Por constructivismo, quiero decir que hay una génesis social de una parte de los esquemas de percepción, pensamiento y de acción que son constitutivos de lo que llamo habitus, y por otra parte estructuras, y en particular de lo que llamo campos y grupos, especialmente de lo que se llama generalmente clases sociales" (Bourdieu 2007b:127).

Las estructuras de dominación masculina no escapan a este esquema de un constructivismo genético o un estructuralismo constructivista, aunque Bourdieu no investigara sustantivamente sobre ellas hasta bien entrada su dilatada carrera investigadora. En La dominación masculina (Bourdieu 2000), la diferencia de género en Bourdieu participa de la óptica crítico-feminista que la entiende desde las relaciones sociales de poder. La pregunta será por qué funciona y se reproduce esa diferencia fundante del orden de unas relaciones de dominación que se pretenden naturales. Pero más que a estas cuestiones, Bourdieu dedica su pensamiento a desvelar la dominación masculina como orden estructurante que se revela en las relaciones más cotidianas entre los sexos, una dominación que, ya de entrada, cifra como violencia simbólica. De este modo sus análisis abren la puerta a una reconsideración teórico-crítica de las relaciones entre los sexos y, precisamente por ello, son de interés para una perspectiva crítico-feminista que se oriente a la transformación de las mismas.

Poder reutilizar y aplicar los conceptos centrales de los análisis bourdieuanos al ámbito de los estudios crítico-feministas y de género, pasa lógicamente por hacerse cargo en un primer momento de algunos de dichos conceptos, de sus significados y alcance. Las herramientas teóricas de Bourdieu para el análisis del mundo social se contraponen a un pensamiento que pretende los hechos sociales como cosas dadas. En lo que hace a los géneros, a hombres y mujeres, su diferencia también es socialmente producida, si bien no por una acción particular de éstos, sino siempre de manera relacional, de tal forma que la praxis social de construcción y deconstrucción genérica no produce individualidades, sino una estructura relacional: “Así, pues, una aprehensión realmente relacional de la relación de dominación entre los hombres y las mujeres tal como se estableció en el conjunto de los espacios y subespacios sociales, es decir, no únicamente en la familia sino también en el universo escolar y en el mundo del trabajo, en el universo burocrático y en el ámbito mediático, conduce a derribar la imagen fantasmal de un «eterno femenino», para resaltar con mayor claridad la persistencia de la estructura de relación de dominación entre los hombres y las mujeres, que se mantiene más allá de las diferencias sustanciales de condición relacionadas con los momentos de la historia y con las posiciones en el espacio social" (Bourdieu 2000, 126-127). 
El carácter relacional de toda estructura social exige conceptos capaces de aprehenderlo. Así, Bourdieu incorpora el concepto de habitus para reflejar la interconexión entre la estructuración social y la acción individual, una interconexión que no se reduce a la aplicación de las normas sociales o las reglas por parte del individuo, sino que expresa la in-corporación de lo social en la producción de la subjetividad. Fuera del dualismo sujeto-objeto, el habitus apela a cómo individuo y mundo interactúan en la praxis social. Y esto implica que las relaciones de dominación no provienen "de fuera", sino que son parte del sistema de clasificación del orden simbólico que atraviesa el mundo social y está incorporado en el individuo. Esto vale también para el dualismo de género, para una estructuración del orden de las prácticas y las representaciones, que no permite entender este habitus solo de manera social, sino también como una historia inscrita en los propios cuerpos y disposiciones.

El habitus es una "estructura estructurante" (Barlösius 2006: 64), que desde el inicio está "generizada" y, a la vez, "generiza" los grupos sociales, los cuerpos, las instituciones, las maneras de la comunicación, etc. El cuerpo es la representación primaria de esta orden de género y es percibido como masculino o femenino desde dentro de las categorizaciones de ese orden: la dualidad y la oposición de los géneros se inscriben primariamente en la interacción con el cuerpo propio y la percepción de los otros cuerpos. Y de aquí que dos tipos de habitus impregnen los cuerpos, a la vez complementarios y opuestos: "La paradoja consiste en que son las diferencias visibles entre el cuerpo femenino y el cuerpo masculino las que, al ser percibidas y construidas de acuerdo con los esquemas prácticos de la visión androcéntrica, se convierten en el garante más indiscutible de significaciones y de valores que concuerdan con los principios de esta visión del mundo; no es el falo (o su ausencia) el fundamento de esta visión, sino que esta visión del mundo, al estar organizada de acuerdo con la división de género relacionales, masculino y femenino, puede instituir el falo, constituido en símbolo de la virilidad, del pundonor (nif) propiamente masculino, y la diferencia entre los cuerpos biológicos en fundamentos objetivos de la diferencia entre los sexos, en el sentido de géneros construidos como dos esencias sociales jerarquizadas" ( Bourdieu 2000, 37).

A partir de esta dualidad naturalizada, las actividades y características de cada género como opuestas se establecen, consideradas en sí mismas, de manera arbitraria, derivándolas no de la diferencia biológica sino de oposiciones sociales: se trata de "una construcción social naturalizada", de un "nomos arbitrario" que se presenta como una ley natural (Bourdieu 2000, 37-38).

Bourdieu parte de lo que llama "la paradoja de la doxa", por la que el orden establecido se reproduce con sus relaciones de dominación, sus desigualdades, sus privilegios y es paradójicamente aceptado incluso en sus condiciones de vida más intolerables. Bourdieu trata de mostrar cómo la violencia simbólica, a la que pertenece la dominación masculina, se reproduce de manera parecida en nuestras sociedades supuestamente avanzadas a cómo ocurre en el caso de la tribu berebere de los cabila. En ambos casos, la arbitrariedad del orden establecido se encubre como un orden que responde a la necesidad natural. Y esta necesidad natural legitima la reproducción, en los cabila y en las sociedades occidentales, del "orden social (que) funciona como una 
inmensa máquina simbólica que tiende a ratificar la dominación masculina en la que se apoya" (Bourdieu 2000, 22).

El esquema binario de clasificaciones arbitrarias (Bourdieu 2000, 20), en el que también se inscribe la dualidad masculino-femenino, constituye el habitus que realiza la dominación de la violencia simbólica entre dominadores (hombres) y dominados (mujeres): "Como producto de la historia de un individuo, en cuanto que experiencia conquistada, el habitus es el resultado de un fuerte trabajo de socialización que se efectúa por medio de la socialización y las instituciones (familia, jardín de infancia, colegio, etc.) y que nos confronta con las formas de pensamiento y de puntos de vista, así como con los esquemas y principios vigentes de la acción y de los principios del juicio y de la valoración. Bourdieu concibe el sujeto como radicalmente socializado desde el inicio, que incorpora el orden social en forma del habitus" (Kremer 2014, p. 36).

La violencia simbólica implica una cierta complicidad por parte de los dominados, ya que su habitus incorpora las acciones que se corresponden con esta forma de relación. Reconocido como legítimo el poder, esto significa que para Bourdieu a esta violencia colaboran las propias mujeres, ya que "las mismas mujeres aplican a cualquier realidad y, en especial, a las relaciones de poder en las que están atrapadas, unos esquemas mentales que son el producto de la asimilación de estas relaciones de poder y que se explican en las oposiciones fundamentales del orden simbólico. Se deduce de ahí que sus actos de conocimiento son, por la misma razón, unos actos de reconocimiento práctico, de adhesión dóxica, creencia que no tiene que pensarse ni afirmarse como tal, y que «crea» de algún modo la violencia simbólica que ella misma sufre"(Bourdieu 2000, 49).

En la sociología de la praxis de Bourdieu, el concepto de habitus juega, pues, un papel central, por cuanto permite dar cuenta a la vez de distintas dimensiones sociales que se interrelacionan: relaciones culturales, económicas, políticas, de dominación, que exigen un pensamiento también relacional. La dimensión simbólica del poder, en este entramado de relaciones, descansa sobre el acuerdo con la dominación de los dominados, porque "[1]os dominados aplican a las relaciones de dominación unas categorías construidas desde el punto de vista de los dominadores, haciéndolas aparecer de ese modo como naturales (...) La violencia simbólica se instituye a través de la adhesión que el dominado se siente obligado a conceder al dominador (por consiguiente, a la dominación) cuando no dispone, para imaginarla o para imaginarse a sí mismo o, mejor dicho, para imaginar la relación que tiene con él, de otro instrumento de conocimiento que aquel que comparte con el dominador y que, al no ser más que la forma asimilada de la relación de dominación, hacen que esa relación parezca natural" (Bourdieu 2000, 50-51). Según esta apreciación, las mujeres participarían de las relaciones de dominación patriarcal, en tanto cuanto reproductoras de tales relaciones como si fueran "el orden natural de las cosas". Se trataría de la incorporación de la dominación masculina que se da en el proceso de socialización con sus diferentes entramados. 
Si esto es así, la posibilidad de subvertir este orden de género pasa por transformar las estructuras mismas de ese orden simbólico dominante. Y ello implica para Bourdieu una revolución simbólica que no solo se limita a una toma de conciencia de la necesidad de tal revolución, sino que transforme las disposiciones y el campo de acción de los agentes.

El concepto de habitus y el de violencia simbólica resultan ser así centrales para analizar las relaciones de dominación, como es el caso de la dominación masculina. Ha habido, no obstante, críticas a la trasposición que realiza Bourdieu del análisis de una cosmología falocéntrica como la de la sociedad de los cabila a las sociedades actuales occidentales. Con ello, se partiría del supuesto de la dominación masculina como orden sociológico y antropológico ahistórico y universal, sin entrar a cuestionarse cuándo y por qué se impone tal dominación (Kröhnert-Othman y Lenz 2002: 167). Esta posición conlleva un peligro de naturalización de esas relaciones de dominación masculina y del orden simbólico consiguiente.

A pesar de esta objeción, el análisis de Bourdieu sobre la dominación masculina abre la puerta a las posibilidades y requisitos para una revolución simbólica, a partir de un análisis del orden simbólico de género dominante y de la estructura social que subyace a este. Pensar y desvelar teóricamente ese orden, sus mecanismos y formas de funcionamiento, es remitirse a una sociología crítica que no se puede entender como independiente de la praxis social.

Es cierto, no obstante, que Bourdieu no se remite a la crítica feminista, ni maneja el corpus teórico desarrollado por esta tanto como sería de desear. Ello hace que sus reflexiones y propuestas no dialoguen con las reflexiones y propuestas que, en especial desde los años 60 y 70 del siglo pasado, han marcado la producción teórica feminista, así como orientado su praxis. En este sentido, se ha hablado de una cierta ignorancia por su parte (Perrot 1998), ya que efectivamente Bourdieu ignora las contribuciones de la teoría feminista al análisis del poder patriarcal y de sus herramientas materiales y simbólicas de dominación, como las que ya hiciera Kate Millett en 1970 al resignificar el concepto de "género" para los análisis crítico-feministas (Millett 1970). A nuestro juicio, sería bueno que Bourdieu hubiera tomado en cuenta este tipo de análisis para incorporar aquellos desarrollos de los mismos que se han orientado hacia la crítica del sistema socio-simbólico que es el patriarcado y que, indudablemente, han guiado al movimiento feminista en una innegable tarea de transformación del mismo en la práctica en muchas sociedades. Reconocer y dialogar con el corpus teórico feminista es, por tanto, algo que se echa de menos en las reflexiones de Bourdieu sobre la dominación masculina.

Para este pensador, la sociología, en tanto que saber reflexivo, tiene que permitir que el sociólogo intervenga con su realidad en la medida en que hace posible que la sociedad reflexione e intervenga sobre sí misma. Si esto es así, entonces hay aquí una propuesta ética y política para orientarse a la posibilidad de llevar a efecto transformaciones sociales desde los discursos teóricos de comprensión de la realidad. Y esta perspectiva teórico-práctica, esta vinculación entre conocimiento y acción o entre teoría y praxis, ha sido algo que ha marcado al pensamiento feminista desde sus inicios como una seña 
de identidad. Es de lamentar que tampoco en La dominación masculina se reconozca el saber feminista como un saber reflexivo que se ajusta a esta perspectiva de ser a la vez arma práctica de transformación de la vida social.

Con todo, a pesar de estas objeciones, el pensamiento de Bourdieu sobre la violencia simbólica, el habitus y la dominación en las relaciones entre los sexos no deja de ser una herramienta útil para el ejercicio de la crítica feminista (Moi 1999). Y no deja de serlo, en particular, si tomamos en cuenta las propias palabras de Bourdieu: "Es indudable que el cambio principal consiste en que la dominación masculina no se haya impuesto con la evidencia de la obviedad. Esto se debe sobre todo al inmenso trabajo crítico del movimiento feminista que, por lo menos en algunas regiones del espacio social, ha conseguido romper el círculo del refuerzo generalizado; tal dominio aparece a partir de ahora, en muchas ocasiones, como algo que hay que defender o justificar, algo de lo que hay que defenderse o justificarse" (Bourdieu 2000: 111). Es decir, que Bourdieu aprecia "los avances que ha hecho el feminismo para romper los círculos de refuerzo del habitus, al cuestionar las estructuras de dominación simbólica, al ampliar el área de lo politizable, logrando introducir en el debate público, en la reivindicación política y en la investigación académica temas que antes estaban destinados a lo femenino o privado" (Gamero Cabrera 2012: 199).

Por tanto, para Bourdieu la dominación masculina constituye el caso paradigmático del ejercicio de la violencia simbólica que persiste en toda la historia de las relaciones sociales. Esa violencia, que presenta la dominación masculina como legitimada por las diferencias anatómicas y biológicas, hace visibles los esquemas de poder que juegan en el campo social, en este caso en el de las relaciones entre los sexos. Al visibilizarlos, Bourdieu se compromete con una crítica que es proyecto de intervención y de transformación de esa relación de dominación y, con ello, su sociología práctica se aúna con los objetivos del movimiento y el pensamiento feministas.

\section{Referencias bibliográficas}

Barlösius, Eva (2006), Pierre Bourdieu. Frankfurt am Main: Campus Verlag.

Bourdieu, Pierre (2000), La dominación masculina. Barcelona: Anagrama (La domination masculine. París: Editions deu Seuil, 1998).

(2007), Cosas Dichas. Barcelona: Editorial Gedisa (Choses dites. París: Les Editions de Minuit, 1987).

Butler, Judith (1990), Gender Trouble. Feminism and the Subversion of Identity. New York: Routledege.

Capdevielle, Julieta (2011), "El concepto de habitus: con Bourdieu y contra Bourdieu", Anduli. Revista Andaluza de Ciencias Sociales 45: 31-45.

Gamero Cabrera, Isabel G. (2012), "Los efectos de la dominación simbólica en el feminismo", Astrolabio. Revista Internacional de Filosofía 13: 189-200.

Kremer, Kathrin (2014), "Pierre Bourdieus männliche Herrschaft in der Frauen und Geschlechterforschung”, en M. Frey, M. Krsizio y G. Jähnert, Männlichkeiten. 
Kontinuität und Umbruch. Berlin: BulletinTexte/Zentrum für transdisziplinäre Geschlechterstudien / Humboldt Universität zu Berlin, Texte, 41, pp. 31-49.

Kröhnert- Othman, Susanne y Ilse Lenz (2002), “Geschlecht und Ethnizität: Kämpfe um Anerkennung und symbolische Regulation", en U.H. Bittlingmayer, R. Eickelpatsch, J. Kastner y C. Rademacher, eds., Theorie als Kampf? Zur politischen Soziologie Pierre Bourdieus. Opladen: Leske+Budrich; pp. 159180.

Millett, Kate (1970), Sexual Politics. Nueva York: Doubleday and Company.

Moi, Toril (1999), “Appropriating Bourdieu: Feminist Theory and Pierre Bourdieu's Sociology of Culture", en T. Moi, What is a Woman? and Other Essays. Oxford: Oxford University Press, pp. 264-299.

Perrot, Michelle (1998), "El feminismo critica: 'lamentamos su ignorancia”, Revista de Estudios de Género. La Ventana. Universidad de Guadalajara 8: pp. 325-32. 